\title{
Microscopic image analysis of elastin network in samples of normal, atherosclerotic and aneurysmatic abdominal aorta and its biomechanical implications
}

\author{
Zbyněk Tonar ${ }^{1,2}$, Stanislav Němeček ${ }^{2}$, Radek Holota ${ }^{2}$, Jitka Kočová ${ }^{1}$, Vladislav Třeška ${ }^{3}$, Jiří Moláček $^{3}$, \\ Tomáš Kohoutek ${ }^{1}$, Šárka Hadravská ${ }^{4}$ \\ ${ }^{1}$ Department of Histology and Embryology, Charles University in Prague, Faculty of Medicine in Pilsen, \\ Czech Republic \\ ${ }^{2}$ New Technologies - Research Centre in Westbohemian Region, University of West Bohemia, Pilsen, Czech \\ Republic \\ ${ }^{3}$ Department of Surgery, University Hospital in Pilsen, Czech Republic \\ ${ }^{4}$ Department of Pathology, Charles University in Prague, Faculty of Medicine in Pilsen, Czech Republic
}

\begin{abstract}
Summary
The aim of our work was to prepare part of the input data for a computational biomechanical model of both the active and passive elements of the tunica media of an aortic aneurysm. We analyzed tissue samples of the anterior wall of the normal, atherosclerotic and aneurysmatic subrenal abdominal aorta. We assessed the proportions of smooth muscle cells, elastin and collagen in histological sections of these samples and studied the morphological characteristics of the elastin network in the tunica media. Selected photomicrographs were studied, representing relatively well preserved areas without artifacts, ruptures, corrupted integrity of the tunica media or total elastinolysis. A new method was introduced for the assessment of structures formed by elastin membranes and fibres, using the fast Fourier transform (FFT) technique. The image was transformed into reciprocal (Fourier) space and the method made use of the fact that the FFT was very sensitive to the orientation distribution of thresholded elastin morphology. The results of this comparative study, obtained from selected samples from 24 patients, revealed that the percentage values of the constituents of the arterial wall can not distinguish between the preserved segments of normal, atherosclerotic or aneurysmatic aorta. The results of the Fourier analysis proved that the FFT provided an efficient method for evaluating cross sections of the elastin membranes and fibres, reflecting their anisotropy. The shape of the power spectrum of elastin was a simple pattern, whose description was quantified by the shape of its polar coordinates histogram. We discuss the methodological difficulties and biomechanical implications of our work as well compare it to other methods of elastin analysis.
\end{abstract}

Keywords: abdominal aortic aneurysm - elastin - image processing - biomechanics

\section{INTRODUCTION}

An aneurysm of the abdominal aorta (AAA) is a serious pathological condition, affecting most often, elderly men. It is defined by the diameter of the subrenal aorta exceeding the normal value by at least $1.5 \times$ in the antero-posterior or latero-lateral direction. The etiology of the most common types of abdominal aortic aneurysm has not been fully elucidated. The pathogenesis of the AAA involves many interconnected and complex processes such as for example, atherosclerosis, inflammation and immune reactions, causing remodelling and degradation of the vessel wall (Wills et al. 1996, Rehm et al. 1998). Prominent morphological features of this vascular disease include changes in 
the arrangement and the proportions of the aortic wall components, such as progressive destruction of the elastin network. The shift in the balance between degradation and resynthesis of the extracellular matrix (Curci et al. 1998) leads to a loss of elastin and to qualitative changes in the collagen fibres. This leads, with or without symptoms, to mechanical inferiority of the aneurysm, and such a weakening results in a further dilatation or even rupture of the aneurysm.

Our aim was to prepare the input data for a computational biomechanical model of both the active and passive elements of the tunica media of the aortic aneurysm. These data were to be based on morphological analysis of the histological samples. This would enable us to carry out in future a numerical experiment modelling the response of the media to intravascular pressure with varying diameters and geometric types of aneurysm.

Microscopical analysis of the aneurysm is not part of its routine clinical diagnostics; it is used rather for retrospective research and is lacking at present a widely accepted methodology. To meet our demand for quantitative data describing the wall of the AAA, we needed to assess the proportions of the smooth muscle cells, elastin and collagen fibres, in the histological sections. In our paper, we shall introduce a procedure for the evaluation of the micrographs of oriented structures by Fourier analysis (Bracewell 1965). We test whether the 2D Fourier transform could be a useful tool for the evaluation of degradation of the elastin network in selected images of the tunica media of the AAA, when compared to the samples of normal and atherosclerotic non-aneurysmatic abdominal aorta.

\section{Fourier analysis}

Conventional image processing techniques operate within "real" space (Russ 1990). We introduce another, mathematical space representing spatial frequencies of the periodic components of the patterns observed in the real structure. Spatial frequency means the number of intersections of the elastin network with an overlaid straight line per unit length. The spatial frequency dimension is $(\text { meter })^{-1}$, i.e. a "reciprocal" meter, and this is why the frequency domain is said to be reciprocal with respect to real space. The image, as observed in real space, is coded as grey level $F(x, y)$ at pixel position $(\mathrm{x}, \mathrm{y})$. The function is expanded into its harmonical components by the Fourier transform. The image is first transformed from the spatial domain to the frequency domain (Fourier space) where a visual representation of the frequency content of the image can be examined. Therefore, the image data displayed in the frequency domain can reveal features not immediately apparent in the spatial domain, where our visual system is often distracted by bright objects and other strong features.

\section{MATERIAL AND METHODS}

The study population encompassed 24 patients. We analyzed tissue samples of the anterior wall of the normal $(n=5), \quad$ atherosclerotic $\quad(n=7)$ and aneurysmatic $(n=12)$ subrenal abdominal aorta. We assessed the proportions of the smooth muscle cells (SMC), elastin and collagen fibres in the area of histological sections $(5-7 \mu \mathrm{m})$ through the wall of the normal aorta and the AAA. We used hematoxylin and eosin; Verhoeff's hematoxylin with green trichrome (Kočová 1970), and aniline blue with Orange G staining. The colour photomicrographs $(3072 \times 2035$ pixels, 24 bit colour depth) were analyzed via the image processing software IMAL developed by Weissar et al. (2001) at the University of West Bohemia in Pilsen, Czech Republic. We identified the components of the tunica media due to their relevance to intervals of RGB values or due to RGB gradient (with variable tolerance). The photomicrographs were selected in order to represent relatively well preserved areas without artifacts, ruptures and without corrupted integrity of the tunica media. We excluded segments of the AAA, where the elastin was totally destroyed. Constant magnification was chosen so that the photographed area could be large enough and free of artifacts in all the samples, and so that the image would not be inclined to reflect local tissue heterogenity.

Selected micrographs of normal, atherosclerotic and aneurysmatic aorta were analyzed by means of 2D FFT (fast Fourier transform). Before the Fourier transform, we performed a thresholding of the elastin in the photomicrograph, using the SW Lucia ${ }^{\circledR}$ 4.51 (Laboratory Imaging Ltd., Prague, Czech Republic). During this process, the use of a selection of narrow ranges of intensity values of the HSI colour model was found more suitable than operating with values of the RGB model. This selection, hopefully comprising the elastin quantitatively, was then segmented (extracted) in order to produce discrete objects. The results of this image segmentation were the monochrome micrographs of the elastin, i.e. encompassing the structure of interest to us.

We developed two programs in the software system MATLAB (The MathWorks, Natrick MA, USA). The first one read an input image with a structure of material under inspection and performed a 2D Fourier Transform (Petrou and Bosdogianni 1999) 
on this data. Then the FFT magnitude was calculated for each point of the image and thresholded into levels 0 or 1. Finally, a new image, composed from the thresholded FFT magnitudes, was saved on hard disk. The second program used the image of the Fourier spectra as an input image and applied the methods for recognition of the degree of elastinolysis. These methods used calculated histograms in polar coordinates, i.e. they summarized the number of white pixels in each direction $\left(0^{\circ}\right.$ to $\left.360^{\circ}\right)$ from the center of the image. Then an enclosed rectangle was constructed and the shape factor was calculated as a ratio of its sides (Holota and Němeček 2002). A minimum of the shape factor was 1 , values near 1 meant small or no deformation.

\section{RESULTS}

In the normal aorta, the tunica media formed a 500$700 \mu \mathrm{m}$ thick layer composed of concentrically arranged anastomosing spiral elastic laminae, which appeared as branched wavy membranes surrounding the 6-18 $\mu \mathrm{m}$ wide spaces containing the fine connective tissue with fibroblasts, collagen (mostly of type III), and smooth muscle cells. The elastin system appeared as a network-like complex of bundles with many sections through the branched elastin bridges interconnecting the neighbouring elastic membranes (Fig. 1). The Fourier transform of these micrographs showed regularity and rotary symmetry (Fig. 2). The parallel system of intact elastin membranes with periodicity in the $y$ axis contributed to the high frequencies. The average shape factor was 97.

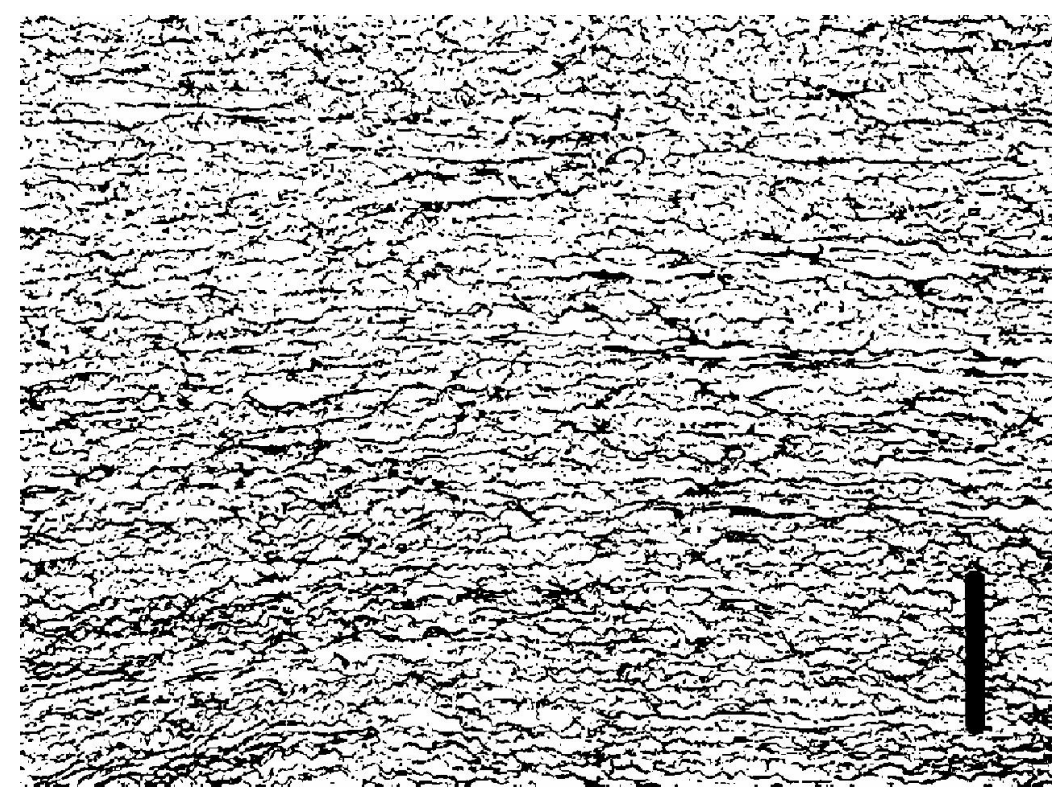

Fig. 1. Image of elastin obtained by thresholding and segmentation of the micrograph of normal aorta, tunica media, bar $=200 \mu \mathrm{m}$

Fig. 2. Frequency spectrum of Fig. 1 
The atherosclerotic abdominal aorta contained quite untouched segments comparable to the normal arterial wall, as well as severely altered parts with elastin loss, where the remainder of the media was repressed beneath the atherosclerotic plaque. In the atherosclerotic aortae, we observed a smoothing and straightening of the elastin membranes (Fig. 3). In the spectrum, it appeared as a persistence of the high frequencies in the $y$ axis representing the periodicity of these mainly parallel structures. The frequencies in other directions were reduced (Fig. 4) and the average shape factor was 72 .

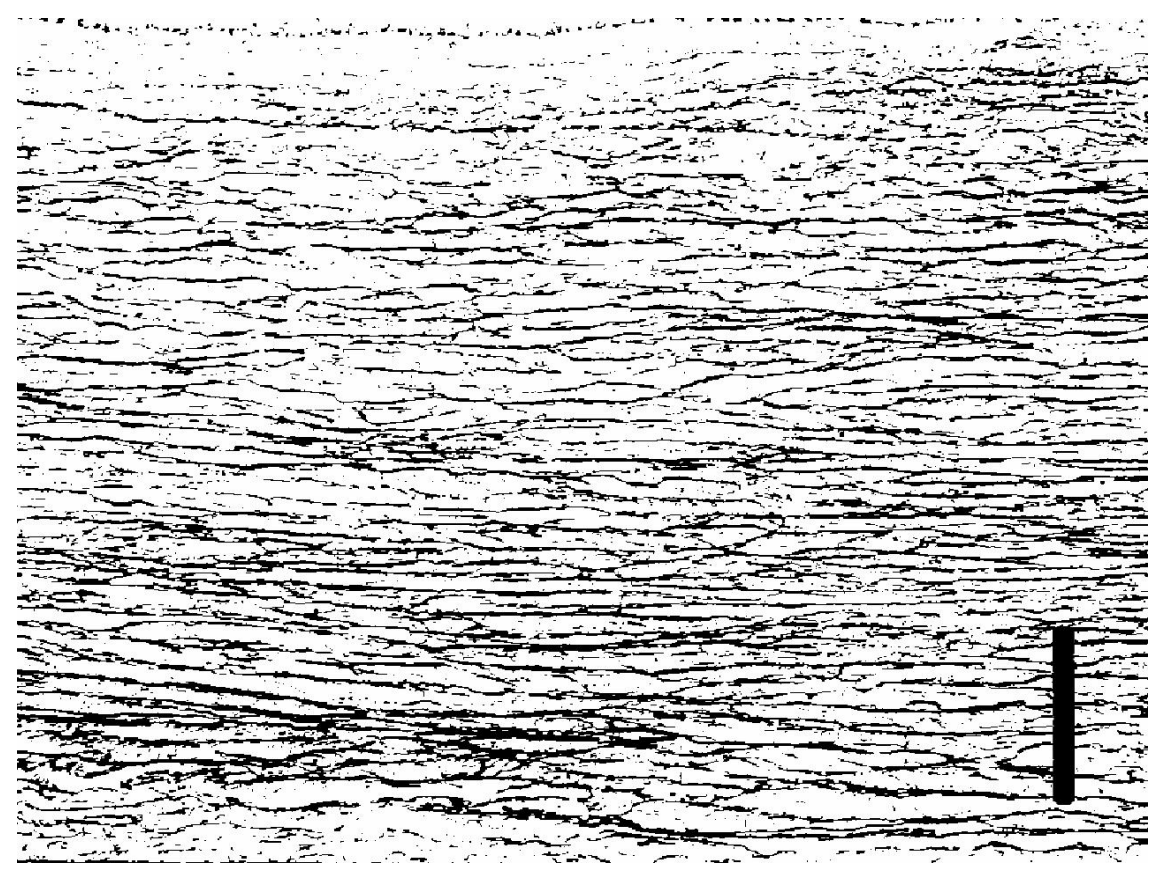

Fig. 3. Image of elastin obtained by thresholding and segmentation of the micrograph of atherosclerotic aorta, well preserved segment of tunica media, bar $=200 \mu \mathrm{m}$ 
In the samples of AAA, there was very much in evidence the disintegration and chaotic rearrangement of the tissue and disappearance of the boundaries between the layers, migration of SMC and inflammatory cells and thickening of the intima by as much as $10 \times$ its normal thickness. The SMC density was decreased and the medial thickness was often reduced. In the tunica media damaged by inflammatory changes, the number of elastin boundaries intersecting the vertical $(y)$ axis was much greater than the number of elastin boundaries intersecting the horizontal $(x)$ axis (Fig. 5): the spatial frequencies in the vertical direction were greater than those in the horizontal direction. The corresponding spectrum was stretched in the vertical direction and squeezed in the horizontal direction (Fig. 6). The fragmentation and absolute diminution of the elastin network led to a fall in the high frequencies in the direction of the arterial circumference ( $y$ axis). The average shape factor was 85 .

The percentage terms of SMC, elastin and collagen are summarized in Table 1 . We calculated the histograms in polar coordinates representing the normal, atherosclerotic and AAA samples (Fig. 7, 8 and 9).

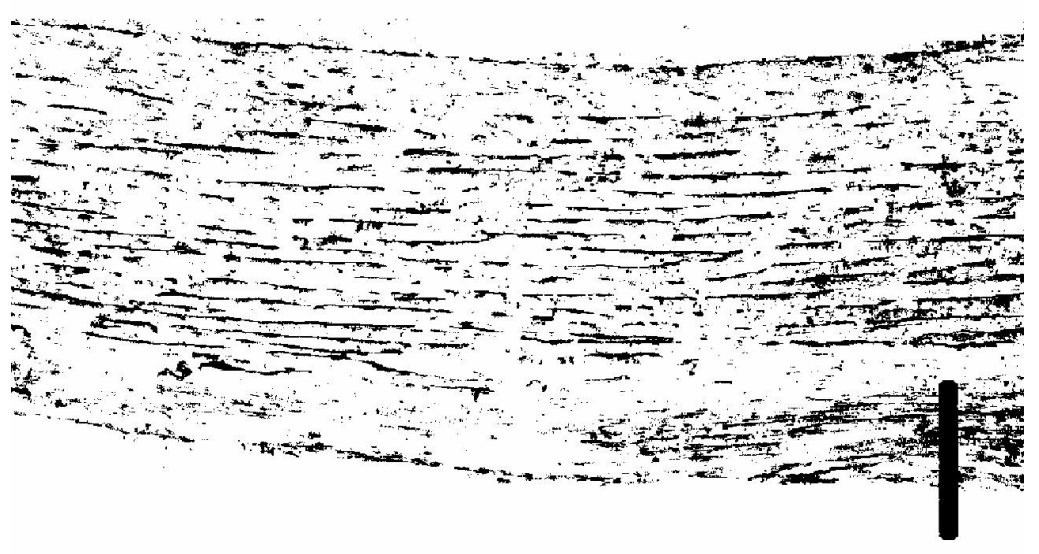

Fig. 5. Image of elastin obtained by thresholding and segmentation of the micrograph of AAA, well preserved segment of tunica media, bar $=200 \mu \mathrm{m}$ 


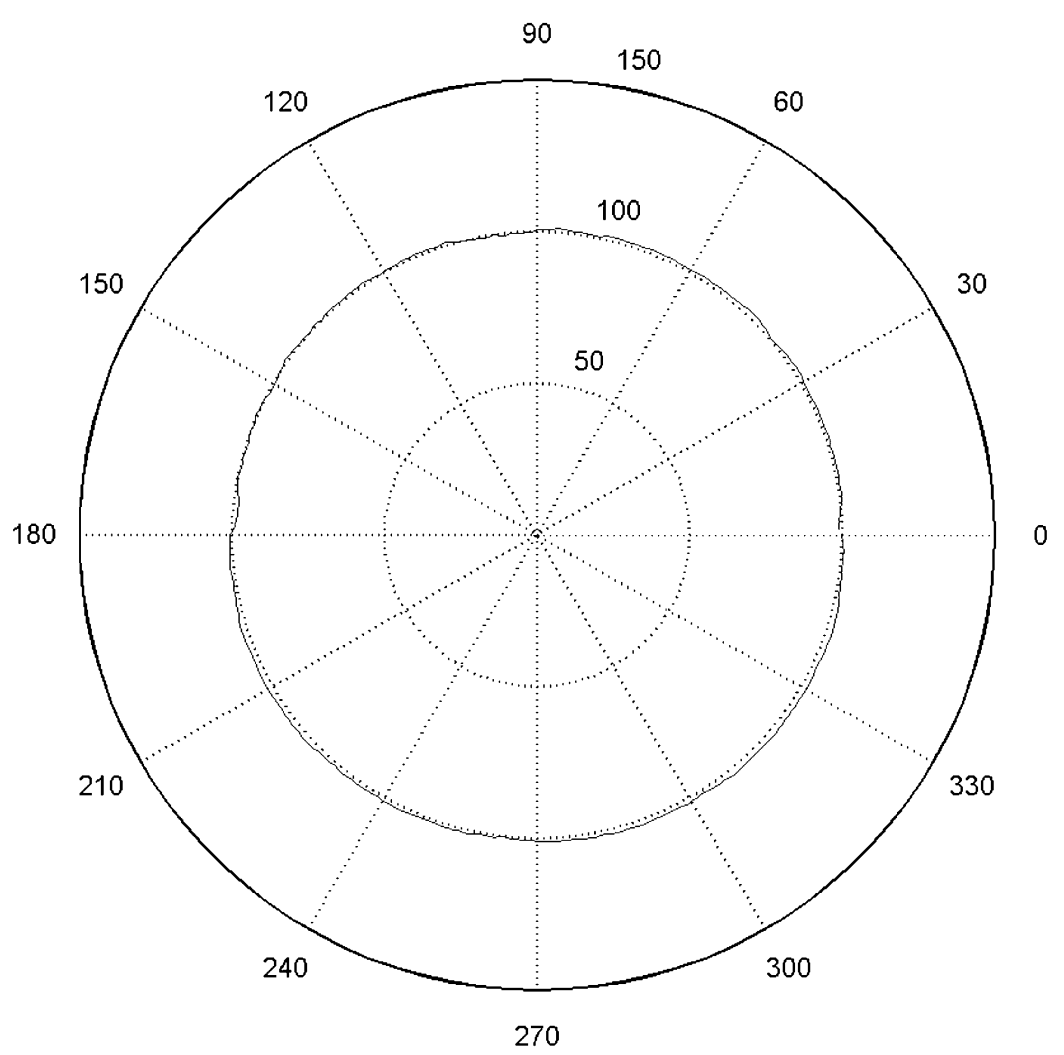

Fig. 7. Histogram in polar coordinates describing the frequency spectrum of Fig. 2 ; shape factor $=0.97$

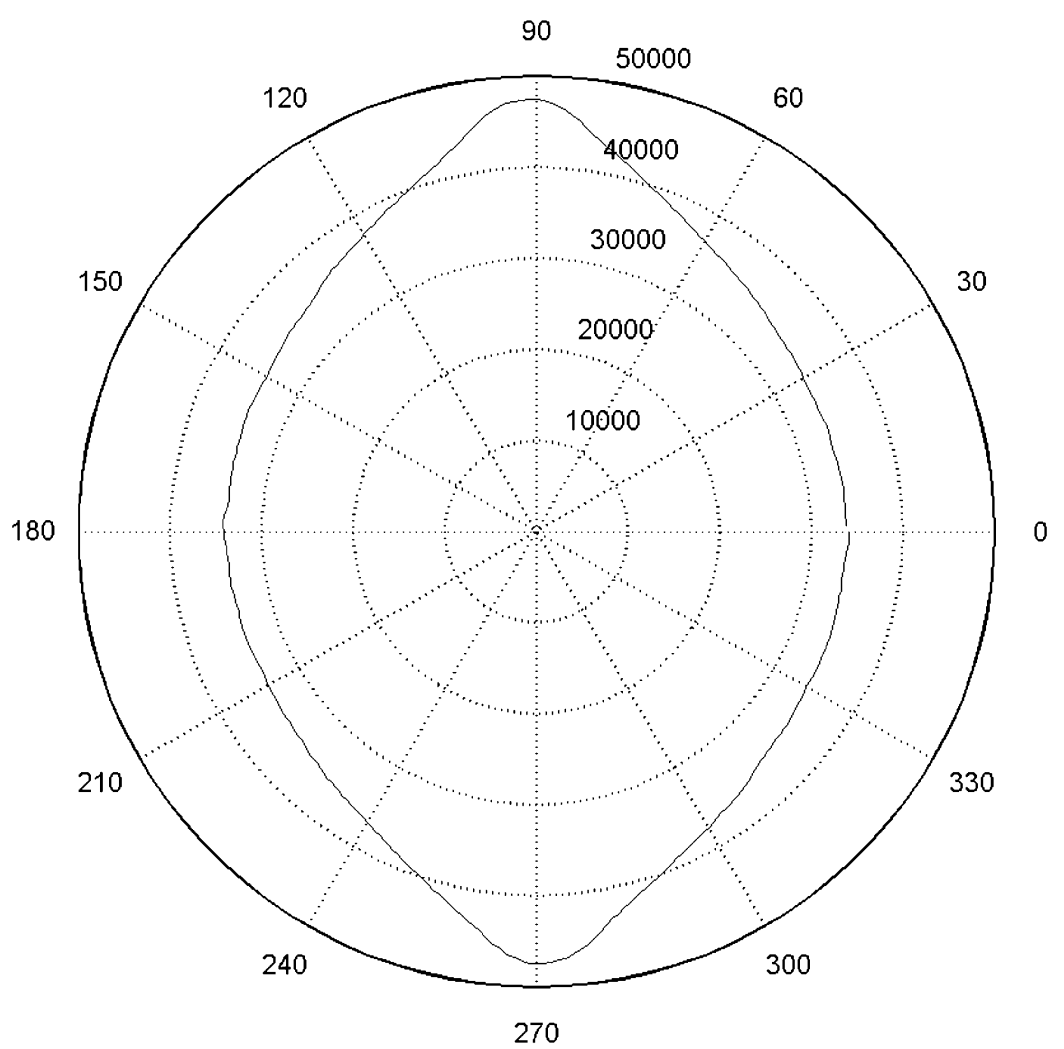

Fig. 8. Histogram in polar coordinates describing the frequency spectrum of Fig. 4 ; shape factor $=0.75$ 


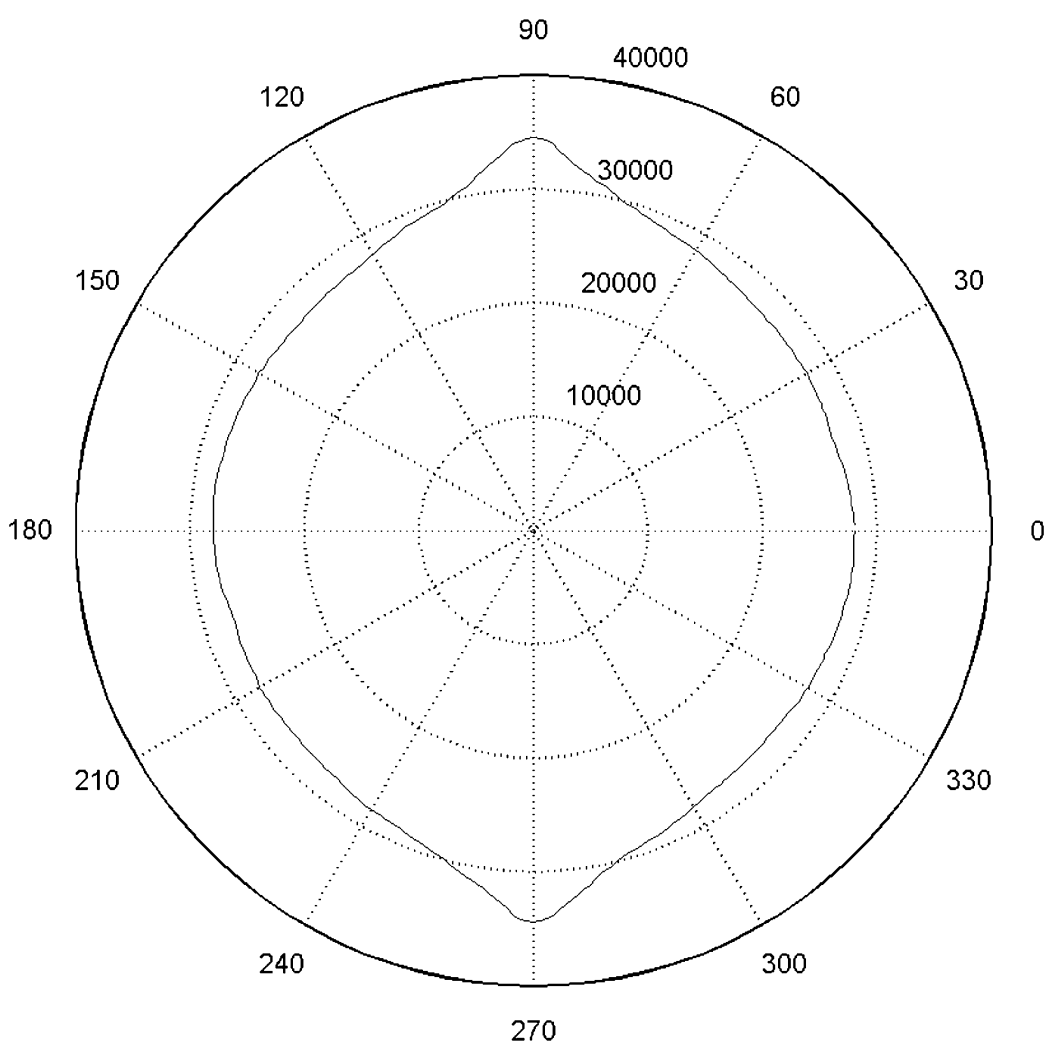

Fig. 9. Histogram in polar coordinates describing the frequency spectrum of Fig. 6 ; shape factor $=0.81$

Table 1. Percentage terms $[\%]$ in the section area through the tunica media

\begin{tabular}{|c|c|c|c|c|}
\hline & \multicolumn{2}{|c|}{ normal aorta } & \multicolumn{2}{|c|}{ AAA } \\
\hline & range of values & $\underline{\text { median }}$ & range of values & median \\
\hline smooth muscle cells & $40-45$ & 44 & $26-29$ & 28 \\
\hline Elastin & $40-60$ & 43 & $0-50$ & 12 \\
\hline collagen I and III & $12-20$ & 18 & $45-51$ & 49 \\
\hline
\end{tabular}

\section{DISCUSSION}

\section{Methodical difficulties}

As it can be seen in the results, the percentage values of the constituents of the arterial wall can not distinguish between the normal, atherosclerotic or aneurysmatic samples of aorta. These data are not specific enough for the groups under study. Moreover, they can achieve considerably different values in the pathologic samples when analyzing 
several micrographs of the neighbouring segments of the same artery. We found these confusing results to be dependent neither on the varying dying properties of the tissue (compensated by the sensitivity and specificity of image segmentation), nor on the magnification used. Even the samples of ruptured AAA can contain some small-sized segments with a minimum of pathological alteration, so that the image processing results are extremely biased by the way in which the photomicrographs are selected. The proper morphological boundaries disappear in the AAA tissue. The loss of rotary symmetry of the wall leads to an extreme variability among the various photomicrographs of the neighbouring segments around the same aneurysm wall. At the same time, we are not able to evaluate these differences by means of statistical methods, because the selected micrographs do not cover the most extensive typical part of the AAA. On the contrary, they have to be rather selective, as explained above in Material and methods. We are aware of the fact that we evaluate some of the segments of the AAA which are not typical for this condition. We try to describe rather the very early changes of the elastin network in relatively well preserved parts of the AAA, while the history of the tissue samples is known to us by definition. A great number of the "typical" (considering the frequency) micrographs on the AAA are not suitable for image processing at all, because of the progressive damage or frequency of the microscopical artifacts.

The Verhoeff's hematoxyline used for quantitative staining of the elastin, stained also the cell nuclei, giving the same colour - from dark violet to black - to both of these types of structures. The formal morphological description of the nuclei is often indistinguishable from that of the cross sections through the elastin fibres. As a result, the elastin thresholding process, although very sensitive, was not specific enough and encompassed also the cross sections through the cell nuclei (belonging mainly to the smooth muscle cells and fibroblasts). However, the samples under study were lacking any obvious discrepancy in nuclei size, number of nuclei per image, and/or nuclear arrangement, because they contained no inflammatory infiltration, signs of angiogenesis, or any deposits of the abnormal extracellular matrix or its proteolysis. This problem represents a possible bias source, but we are convinced it did not distort the results of the FFT. The problem of varying staining properties of the elastin required an adaptive thresholding, i.e. the modulation of the contrast mechanism defining the structure of interest to us.

In order to comply with its function as a whole, the elastin network has to maintain its degree of cross linking, i.e. the quality and quantity of the interconnections among the neighbouring fenestrated membranes of the tunica media. The disappearance of the elastin observed with light microscopy belongs rather to the late signs of its advanced deterioration, when most of its function capabilities are already lost. Our technique sets out to evaluate the degree of fragmentation and straightening of the components of the elastin network, as visible on the 2D sections through the arterial wall, which should be a better correlate of the progression of aneurysmatic changes than the percentage of elastin in the arterial wall. Of course, it can be disputable as to what extent the evaluation of the individual 2D tissue slides can predicate the elastin network as a 3D structure. Furthermore, because of the scale factor, we always analyzed only a small part of the whole arterial circumference.

\section{Other methods of elastin analysis}

From a pathologist's point of view, there are several phenomena to be combined in order to evaluate the alteration of the AAA wall. These can be for example the type and morphometry of the atherosclerotic plaques (Zarins et al. 2001) and their calcification (Matsushita et al. 2000), erosion and ulceration, presence and size of mural thrombi (Patel et al. 1994, Hallingbye and Kane 2002), angiogenesis (Satta et al. 1998, Thompson et al. 1996, Holmes et al. 1995, Kobayashi et al. 2002), haemorrhages and dissection (Farber et al. 2002), inflammatory infiltrates (Jones et al. 2001, Bonamigo et al. 2002) and both local and plasma activity of the cytokines (Třeška et al. 1998, Rohde et al. 1999). The affection of the matrix turnover through the modulation of the matrix metalloproteinase expression is one of the cardinal factors in the evolution and progression of AAA (Davis et al. 2001), together with the failure of the antiproteolytic system (tissue inhibitor of matrix metalloproteinase), as demonstrated by Allaire et al. (2002), Silence et al. (2002) and Nollendorfs et al. (2001). The quantitative immunohistochemical assessment of macrophages and $\mathrm{T}$ lymphocytes infiltration is meaningful as well, because these cells produce cell death-promoting proteins (Henderson et al. 1999) contributing to the elimination of smooth muscle cells which are the source of elastin and collagen. With increasing age, the abundance of elastin associated microfibrils decreases, even in the normal aorta (Godfrey et al. 1993). However, we suppose the degradation rate of elastin to be the most suitable and meaningful of the potential objects of image processing, along with the analysis of immunohistochemically marked inflammatory cells. Where there are various microanalytical techniques available, it is often desirable to combine them in a complementary way to maximize the 
information available from the specimen. Avolio et al. (1998) characterized the structure of elastin in terms of textural features, quantifying their alterations by fractal parameters and other stereological methods. The use of the confocal laser scanning microscopy represents another effective method for description of the three-dimensional arrangement of arterial elastin (Carvalho and Taboga 1996). Carmo et al. (2002) confirmed a decreased elastin content in the AAA and a concurrent increase of collagen (as proved by HPLC and amino acid analysis). The latter study proved the content of desmosines and isodesmosines to be a useful marker of elastin cross-links assessment.

\section{The role of elastin in AAA development}

We propose the following hypothesis of the mechanical pathogenesis of the AAA, consistent with our observation: The inflammatory changes damage inter alia the elastin in the thickest arterial layer, i.e. tunica media. The wall is no longer elastic enough to be able to accumulate the energy of the pressure amplitude during the systole and to return this energy back to the blood. At the same time, mechanical demands on the arterial wall do not reduce. On the contrary, the pressure load of the fibrillar extracellular matrix rises, together with the phenotypic conversion of the smooth muscle cells to their metabolic type with a reduced contractile capability. The decreased density of smooth muscle cells causes a weakening of the paracrine mechanisms protecting the artery wall homeostasis (Allaire et al. 2002). Due to the reduced compliance of the artery (together with the hypertension), the increased integrated continual pressure causes the long term remodellation of the wall, resulting in a further expansion of the aneurysm.

\section{The FFT analysis of the elastin - benefits and prospects}

Mechanical deformation of the arterial wall, as well as biochemical processes, may result in disintegration of the fine interconnecting elastin fibres in one direction and in straightening of the persistent elastic membranes in the other direction. That is why the spatial frequencies diverge in different directions: they are lower in the direction in which the fibres have been relatively elongated and higher in the direction in which they have been "compressed". As a consequence of this, the Fourier transform of the micrograph becomes anisotropic and elliptical. On the contrary, in the case of the equiaxial elastin network, the spatial frequencies are the same in all directions and the Fourier transform of the micrograph of such a structure is isotropic, i.e. circular. The anisotropy of the Fourier transform
$\mathrm{F}(\mathrm{u}, \mathrm{v})$ of the micrograph under analysis reflects the anisotropy of the structure as perceived directly on the micrograph of the structure $f(x, y)$. But the Fourier spectrum $\mathrm{F}(\mathrm{u}, \mathrm{v})$ (or the power spectrum $\left.|F(u, v)|^{2}\right)$ is much simpler than the image $f(x, y)$, and therefore the evaluation of the Fourier transform of images is easier and gives more detailed and exact information on deformed structures than the evaluation of the image of the micrograph (Saleh and Teich 1991).

In order to test the applicability and universality of the FFT for the description of elastin morphology in further studies, we suggest a comparison of the FFT findings in normal aorta of the adult and infantile patients, where the effect of different geometry and elastin content should be demonstrated. It could be also tested, whether the method presented in this study is able to describe the difference between the elastin in the thoracic and abdominal aorta. It is well known that in the thoracic part of the aorta, the percentage terms of elastin in the media and complexity of its network exceeds these parameters in the abdominal part. By comparing the longitudinal and transversal section through neighbouring segments of the same aorta, the FFT could also be able to describe the anisotropy of the elastin network in the third dimension. The method presented could also prove whether the thickness of the elastin lamellar units can differ in the inner and outer zones of the tunica media, building a morphologic gradient across the media. Concerning the AAA modelling, it would be useful to prove whether the dilatation of the aneurysm correlates with the length and pattern characteristic of the section through the elastin network and whether there are any dependencies between the length of the fibres and their shape. In our further work, the dependence of the result on the magnification used should be tested, as well as the variability of the results among different parts of the same tissue sample and of the same photomicrograph, the reproducibility and the intraand interobserver variation.

\section{Biomechanical implications}

The current methods of AAA detection and followup belong to medical imaging techniques. Factors determining whether surgery is necessary are the size and expansion rate of the AAA as well as the age and health of the patient. Therefore we can see the possible benefit of our method in the field of AAA modelling rather than in clinical practice, although we do not suppose the changes of mechanical properties of the AAA to be a simple function of the changing morphology of the elastin structure. The computational modelling of the AAA wall distensibility could be useful, because this 
parameter can provide (together with diastolic blood pressure and diameter) a quite successful assessment of the AAA rupture risk, as has been proved recently (Wilson et al. 2003). The consequences of our observation on the background of the biomechanical modelling of the aortic wall are as follows: It has to be considered that the metabolically altered fibres and cells are not mechanically analogous to their physiological equivalents. The soft tissue model should be based on the mixture theory approach and the microstructure of the tunica media should be characterized by volume fractions and preferential orientation of the basic components involved: the active fibres representing the bundles of muscle cells, two types of passive fibres corresponding to the collagen/elastin fibres, and the matrix. In the aneurysmatic and atherosclerotic wall the mechanical importance of smooth muscle cells is suppressed, as they are minor in comparison with other components (collagen, elastin) and mostly transformed to the metabolic phenotype, so that their activation can be regarded as constant. As can be seen, the model will be focused on the mechanics of the tunica media only. Because of insufficient data, there will not be implemented the biological phenomena such as innervation, endothelial dys/function, mediators, inflammatory remodelling, presence of thrombi and calcification in this mechanical model.

We can conclude that a new technique was used to determine the degree of degradation of elastin by means of the 2D fast Fourier transform of its thresholded photomicrograph. This approach proved itself to be a useful tool when searching for a universal method of elastin network description, but its reliability has to be further tested. Because of the many possible methodical pitfalls and variability of the samples of AAA, we found this method to be unsuitable for routine automatized image processing. It requires a thoughtful selection of the input photomicrographs of the arterial wall and a careful reading of the output data. Under such conditions it promises to enable us to differentiate both the successive stages of progressive elastinolysis and deformation of the arterial wall very sensitively.

\section{ACKNOWLEDGEMENTS}

This paper is based upon work sponsored by the Ministry of Education of the Czech Republic under research and development project LN00B084. Partially supported by the project CEZ J13/98:1114000001.

Received $9^{\text {th }}$ April 2003.

Published online $12^{\text {th }}$ June 2003.

\section{REFERENCES}

Allaire E., B. Muscatelli-Groux, C. Mandet, A.M. Guinault, P. Bruneval, P. Desgranges: Paracrine effect of vascular smooth muscle cells in the prevention of aortic aneurysm formation. J. Vasc. Surg. 36: 1018-1026, 2002.

Avolio A., D. Jones, M. Tafazzoli-Shadpour: Quantification of alterations in structure and function of elastin in the arterial media. Hypertension 32: 170-175, 1998.

Bonamigo T.P., C. Bianco, M. Becker, F. Puricelli: Inflammatory aneurysms of infra-renal abdominal aorta. A case-control study. Minerva Cardioangiol. 50: 253-258, 2002.

Bracewell R.N.: The Fourier Transformation and its Applications, McGraw-Hill Book Company, New York 1965, pp. 640.

Carmo M., L. Colombo, A. Bruno, F.R. Corsi, L. Roncoroni, M.S. Cuttin, F. Radice, E. Mussini, P.G. Settembrini: Alteration of elastin, collagen and their cross-links in abdominal aortic aneurysms. Eur. J. Vasc. Endovasc. Surg. 23: 543-549, 2002.

Carvalho de H.F. and S.R. Taboga: Fluorescence and confocal laser scanning microscopy imaging of elastic fibers in hematoxylin-eosin stained sections. Histochem. Cell. Biol. 106: 587-592, 1996.

Curci J.A., S. Liao, M.D. Huffman, S.D. Shapiro: Expression and localization of macrophage elastase (matrix metalloproteinase-12) in abdominal aortic aneurysms. J. Clin. Invest. 102: 1900-1910, 1998.

Davis V.A., R.N. Persidskaia, L.M. Baca-Regen, N. Fiotti, B.G. Halloran, B.T. Baxter: Cytokine pattern in aneurysmal and occlusive disease of the aorta. J. Surg. Res. 101: 152-156, 2001.

Farber A., W.H. Wagner, D.V. Cossman, J.L. Cohen, D.B. Walsh, M.F. Fillinger, J.L. Cronenwett, S.R. Lauterbach, P.M. Levin: Isolated dissection of the abdominal aorta: clinical presentation and therapeutic options. J. Vasc. Surg. 36: 205-210, 2002.

Godfrey M., P.A. Nejezchleb, G.B. Schaefer, D.J. Minion, Y. Wang, B.T. Baxter: Elastin and fibrillin mRNA and protein levels in the ontogeny of normal human aorta. Connect. Tissue Res. 29: 61-69, 1993.

Hallingbye T.M. and J.J. Kane: Examining a CT scan of an abdominal aortic aneurysm. Part 1: Measuring the thrombus to aneurysm ratio using MatLab. Biomed. Sci. Instrum. 38: 375380, 2002.

Henderson E.L., Y.J. Geng, G.K. Sukhova, A.D. Whittemore, J. Knox, P. Libby: Death of smooth muscle cells and expression of 
mediators of apoptosis by $\mathrm{T}$ lymphocytes in human abdominal aortic aneurysms. Circulation 99: 96-104, 1999.

Holmes D.R., S. Liao, W.C. Parks, R.W. Thompson: Medial neovascularisation in abdominal aortic aneurysms: a histopathologic marker of aneurysmal degeneration with pathophasiologic implications. J. Vasc. Surg. 21: 761-771, 1995.

Holota R. and S. Němeček: Recognition of oriented structures by 2D Fourier transform. - In Pinker J. (ed.): Applied Electronics 2002, University of West Bohemia, Pilsen 2002, pp. 88-92.

Jones K.G., D.J. Brull, L.C. Brown, M. Sian: Interleukin-6 (IL-6) and the prognosis of abdominal aortic aneurysms. Circulation 103: 2260-2265, 2001.

Kobayashi M., J. Matsubara, M. Matsushita, N. Nishikimi, T. Sakurai, Y. Nimura: Expression of angiogenesis and angiogenic factors in human aortic vascular disease. J. Surg. Res. 106: 239-245, 2002.

Kočová J.: Overall staining of connective tissue and the muscular layer vessels. Folia Morphol. 18: 293-295, 1970.

Matsushita M., N. Nishikimi, T. Sakurai, Y. Nimura: Relationship between aortic calcification and atherosclerotic disease in patients with abdominal aortic aneurysm. Int. Angiol. 19: 276-279, 2000.

Nollendorfs A., T.C. Greiner, H. Nagase, B.T. Baxter: The expression and localization of membrane type-1 matrix metalloproteinase in human abdominal aortic aneurysms. J. Vasc. Surg. 34: 316-322, 2001.

Patel H., M. Krishnamoorthy, R.A. Dorazio, J. Abu Dalu, R. Humphrey, J. Tyrell: Thrombosis of abdominal aortic aneurysms. Am. Surg. 60: 801-803, 1994.

Petrou M. and P. Bosdogianni: Image Processing: The Fundamentals. Willey, New York 1999, pp. 354.

Rehm J.P., J.J. Grange, B.T. Baxter: The formation of aneurysm. Seminars in Vascular Surgery 11: 193-202, 1998.

Rohde L.E., L.H. Arroyo, N. Rifai, M.A. Creager, P. Libby, P.M. Ridker, R.T. Lee: Plasma concentrations of interleukin- 6 and abdominal aortic diameter among subjects without aortic dilatation. Arterioscler. Thromb. Vasc. Biol. 19: 1695-1699, 1999.

Russ J.C.: Computer-assisted microscopy. Plenum Press, New York 1990, pp. 466.

Saleh B.E.A. and M.C. Teich: Fundamentals of Photonics. Willey, New York 1991, pp. 992.

Satta J., Y. Soini, M. Mosorin, T. Juvonen: Angiogenesis is associated with mononuclear inflammatory cells in abdominal aortic aneurysms. Annales Chirurgiae et Gynecologiae 87: 40-42, 1998.

Silence J., D. Collen, H.R. Lijnen: Reduced atherosclerotic plaque but enhanced aneurysm formation in mice with inactivation of the tissue inhibitor of metalloproteinase-1 (TIMP1) gene. Circ. Res. 90: 897-903, 2002.

Thompson M.M., L. Jones, A. Nasim, R.D. Sayers: Angiogenesis in abdominal aortic aneurysms. Eur. J. Vasc. Endovasc. Surg. 11: 464-469, 1996.

Třeška V., O. Topolčan, P.W. Wenham, L. Pecen: Metabolism of cytokines in abdominal aortic aneurysms. Rozhl. chir. 77: 225-229, 1998.

Weissar P., J. Fiřt, Z. Tonar: IMAL - the software for image analysis of the histology of normal and aneurysmatic aorta. In Pinker J. (ed.): International Conference Applied Electronics 2001, University of West Bohemia, Pilsen 2001, pp. 260-261.

Wills A., M.M. Thompson, M. Crowether, R.D. Sayers: Pathogenesis of abdominal aortic aneurysms - cellular and biochemical mechanisms. Eur. J. Vasc. Endovasc. Surg. 12: 391-400, 1996.

Wilson K.A., A.J. Lee., P.R. Hoskins, F.G. Fowkes, C.V. Ruckley, A.W. Bradbury: The relationship between aortic wall distensibility and rupture of infrarenal abdominal aortic aneurysm. J. Vasc. Surg. 37: 112-117, 2003.

Zarins C.K., C. Xu, S. Glagov: Atherosclerotic enlargement of the human abdominal aorta. Atherosclerosis 155: 157-164, 2001.

\section{* Address:}

Zbyněk Tonar, Department of Histology and Embryology, Charles University in Prague, Faculty of Medicine in Pilsen, Karlovarská 48, 30166 Pilsen, Czech Republic; e-mail: zbynek.tonar@lfp.cuni.cz 